\title{
A new type of three-dimensional deep-water wave of permanent form
}

\author{
By PHILIP G. SAFFMAN
}

Applied Mathematics, California Institute of Technology, Pasadena, California 91125

\section{AND HENRY C. YUEN}

Fluid Mechanics Department, TRW Defense and Space Systems Group, Redondo Beach, California 90278

(Received 7 January 1980 and in revised form 14 April 1980)

A new class of three-dimensional, deep-water gravity waves of permanent form has been found using an equation valid for weakly nonlinear waves due to Zakharov (1968). These solutions appear as bifurcations from the uniform two-dimensional wave train. The critical wave heights are given as functions of the modulation wave vector. The three-dimensional patterns may be skewed or symmetrical. An example of the skewed wave pattern is given and shown to be stable. The results become exact in the limit of very oblique modulations.

\section{Introduction}

The existence and properties of finite-amplitude, deep-water gravity waves have been of considerable interest since the initial study by Stokes (1847). Properties of two-dimensional, uniform waves of permanent form have been found from the weakly nonlinear Stokes waves up to the wave of greatest height (see, for example, Schwartz 1974). Recently Chen \& Saffman (1980) showed numerically that the continuation of the Stokes waves can bifurcate at large amplitude into non-uniform, two-dimensional steady waves. Further results dealing with bifurcations associated with long wave modulation have been obtained by Saffman (1980).

In this paper, we consider the problem of bifurcation of a uniform, two-dimensional wave train into three-dimensional steady waves of permanent form. This problem is considerably more difficult than the two-dimensional case, since the resources of function theory are not readily extendable. However, it is known that long-wave modulations of weakly nonlinear waves to both two- and three-dimensional disturbances are well described by an integro-differential equation first derived by Zakharov (1968) (see Crawford et al. 1981). This equation lends itself to the study of bifurcation into three-dimensional waves, and will be shown to imply that at an amplitude dependent on the wavelength and direction of the modulation, the uniform wave train can bifurcate into a skewed or a symmetric wave form. The critical amplitude approaches zero as the modulation becomes more and more oblique. In this limit, the 
results obtained here will be accurate descriptions of the exact water-wave behaviour. It is notable that even for the two-dimensional bifurcations, which occur at very large amplitude, the Zakharov approximation still gives good qualitative and reasonable quantitative agreement with the numerical results from the exact equation.

We shall also exhibit examples of free surface profiles corresponding to a skewed bifurcated solution, and demonstrate that it is stable to infinitesimal perturbations.

\section{The evolution equation and 'Stokes wave'}

The equation governing the evolution of three-dimensional weakly nonlinear, deepwater gravity waves has been shown by Zakharov (1968) to be

$$
\begin{array}{r}
i \frac{\partial B}{\partial t}(\mathbf{k}, t)=\int_{-\infty}^{\infty} \iint T\left(\mathbf{k}, \mathbf{k}_{1}, \mathbf{k}_{\mathbf{2}}, \mathbf{k}_{\mathbf{3}}\right) \delta\left(\mathbf{k}+\mathbf{k}_{\mathbf{1}}-\mathbf{k}_{\mathbf{2}}-\mathbf{k}_{\mathbf{3}}\right) \exp \left\{i \left[\omega(k)+\omega\left(k_{\mathbf{1}}\right)\right.\right. \\
\left.\left.-\omega\left(k_{2}\right)-\omega\left(k_{3}\right)\right] t\right\} B^{*}\left(\mathbf{k}_{1}, t\right) B\left(\mathbf{k}_{\mathbf{2}}, t\right) B\left(\mathbf{k}_{\mathbf{3}}, t\right) d \mathbf{k}_{\mathbf{1}} d \mathbf{k}_{\mathbf{2}} d \mathbf{k}_{\mathbf{3}},
\end{array}
$$

where $\mathbf{x}=(x, y)$, the free surface $\eta(\mathbf{x}, t)$ to lowest order is related to $B(\mathbf{k}, t)$ by

$\eta(\mathbf{x}, t)=\frac{1}{2 \pi} \int \frac{k^{\frac{1}{2}}}{2^{\frac{1}{2}} g^{\frac{1}{4}}}\left[B(\mathbf{k}, t) \exp \{i[\mathbf{k} \cdot \mathbf{x}-\omega(k) t]\}+B^{*}(\mathbf{k}, \mathbf{t}) \exp \{-i[\mathbf{k} \cdot \mathbf{x}-\omega(k) t]\}\right] d \mathbf{k}$,

where $g$ is the acceleration due to gravity, $\mathbf{k}$ is the surface wave vector, $k=|\mathbf{k}|$ is its magnitude, and $\omega(k)$ is the frequency from linear theory,

$$
\omega(k)=(g k)^{\frac{1}{2}} .
$$

The interaction coefficient $T\left(\mathbf{k}, \mathbf{k}_{1}, \mathbf{k}_{2}, \mathbf{k}_{3}\right)$, which is real, was first given by Zakharov (1968), and can be found in Crawford, Saffman \& Yuen (1980) with minor algebraic correction.

We are interested in solutions corresponding to three-dimensional modulations imposed on an otherwise uniform two-dimensional wavetrain which propagates in the $\mathbf{e}_{1}$ direction $\left[\mathbf{e}_{1}=(1,0), \mathbf{e}_{2}=(0,1)\right]$ with unit wavenumber. Thus we take

where

$$
B(\mathbf{k}, t)=\sum_{1} \tilde{a}_{1}(t) \delta(\mathbf{k}-1) e^{i \omega(k) t},
$$

$$
1=l \mathbf{e}_{1}+m p \mathbf{e}_{1}+n q \mathbf{e}_{2},
$$

$m, n$ are positive or negative integers, and $\mathbf{p}=(p, q)$ is a pair of real numbers. The undisturbed wave corresponds to $l=1, m=0, n=0$.

The approximations inherent in arriving at equation (1) lead to the neglect of interactions between harmonics of the undisturbed fundamental and the modulations. There is therefore a class of waves in which $l=1$ for all modes and for which the mode $\mathbf{l}$ is uniquely labelled by an integer pair $\mathbf{m}=(m, n)$ for a given fundamental modulation p. For this class, equation (4) can then be written, for a given choice of $\mathbf{p}$, as

$$
B(\mathbf{k}, t)=\sum_{\mathbf{m}} \tilde{a}_{\mathbf{m}}(t) \delta(\mathbf{k}-\mathbf{1}) e^{i \omega(k) t},
$$

where

$$
\mathbf{1}=\mathbf{e}_{\mathbf{1}}+\mathbf{m}: \mathbf{p} \equiv \mathbf{e}_{\mathbf{1}}+m p \mathbf{e}_{\mathbf{1}}+n q \mathbf{e}_{\mathbf{2}}
$$


Substitution of expression (6) into equation (1) gives

$$
i \frac{d \tilde{a}_{\mathbf{m}}}{d t}-|1| \frac{1}{2} \tilde{a}_{\mathbf{m}}=\sum_{\mathbf{m}+\mathbf{i}=\mathbf{j}+\mathbf{k}} T_{\mathbf{m}, \mathbf{i}, \mathbf{j}, \mathbf{k}} \tilde{a}_{\mathbf{i}}^{*} \tilde{a}_{\mathbf{j}} \tilde{a}_{\mathbf{k}}
$$

where $g$ is taken to be unity, and we use the notation

$$
T_{\mathrm{m}, \mathbf{i}, \mathbf{j}, \mathrm{k}} \equiv T[\mathbf{1}(\mathbf{m}), \mathbf{1}(\mathbf{i}), \mathbf{1}(\mathbf{j}), \mathbf{1}(\mathbf{k})]
$$

We now consider solutions of this class for which

$$
\eta(\mathbf{x}, t)=\eta(x-C t, y),
$$

which describe surface profiles propagating without change of shape at a constant speed $C$ in the $\mathbf{e}_{\mathbf{1}}$ direction. The two-dimensional Stokes wave corresponds to a solution which has no dependence on $y$. We shall demonstrate the existence of extensions of this solution which describe steady waves of permanent form with three-dimensional structure. This involves finding solutions of (8) in the form

$$
\tilde{a}_{\mathrm{m}}=a_{\mathrm{m}} \exp \{-i 1(\mathrm{~m}) \cdot \mathrm{C} t\}=a_{\mathrm{m}} \exp \{-i(1+m p) C t\}
$$

where $\mathbf{C}=(C, 0)$, and the $a_{\mathrm{m}}$ are independent of $t$.

In this notation, the uniform, two-dimensional solution (Stokes wave) is

$$
\left.\begin{array}{cc}
a_{\mathrm{m}}^{*}=a_{\mathrm{m}}=a(\text { const. }), & m=0, \\
a_{\mathrm{m}}=0, & m \neq 0,
\end{array}\right\}
$$

and equation (8) is obviously satisfied by

It can be verified that

$$
C=1+T_{0,0,0,0} a^{2}
$$

$$
T_{0,0,0,0}=\frac{1}{4 \pi^{2}}
$$

and the solution (12)-(13) agrees with the results of Stokes (1847). Note that from equation (2) the wave amplitude is $a /(2 \pi)^{\frac{1}{2}}$.

Substituting (11) into (8) and its complex conjugate, we obtain the evolution equations, relative to a frame moving with speed $C$, of modulations associated with a modulation wave vector $\mathbf{p}$,

$$
\begin{aligned}
& i \frac{d a_{\mathrm{m}}}{d t}=\left\{|\mathbf{1}(\mathbf{m})|^{\frac{1}{2}}-(1+m p) C\right\} a_{\mathrm{m}}+\sum T_{\mathrm{m}, \mathrm{i}, \mathrm{j}, \mathbf{k}} \hat{a}_{\mathbf{i}} a_{\mathbf{j}} a_{\mathbf{k}}, \\
& i \frac{d \hat{a}_{\mathrm{m}}}{d t}=-\left\{|\mathbf{1}(\mathbf{m})|^{\frac{1}{2}}-(1+m p) C\right\} \hat{a}_{\mathrm{m}}-\sum T_{\mathrm{m}, \mathrm{i}, \mathrm{j}, \mathbf{k}} a_{\mathrm{i}} \hat{a}_{\mathrm{j}} \hat{a}_{\mathbf{k}},
\end{aligned}
$$

where physical validity requires the satisfaction of the relation

$$
\hat{a}_{\mathrm{m}}=a_{\mathrm{m}}^{*}
$$

and the members of the integer pairs $m, \mathbf{i}, \mathbf{j}, \mathbf{k}$ run from $-\infty$ to $\infty$. It proves to be convenient in analysing the stability of steady solutions to allow (16) to be violated by the infinitesimal perturbations, but it is always satisfied for steady waves.

Equations (15) can be written symbolically as

$$
i \mathbf{u}_{t}=M(\mathbf{u}, C)
$$


where $M$ is a nonlinear cubic operator and

$$
\mathbf{u}=\left[\left\{a_{\mathbf{m}}\right\} ;\left\{\hat{a}_{\mathbf{m}}\right\}\right] .
$$

is a member of a Hilbert space with norm

$$
\|\mathbf{u}\|^{2}=\sum_{\mathbf{m}}\left(\hat{a}_{\mathrm{m}} \hat{a}_{\mathbf{m}}^{*}+a_{\mathbf{m}} a_{\mathbf{m}}^{*}\right)
$$

and an obvious definition of the inner produet.

We now proceed to investigate steady solutions of (17) which appear from bifurcation of the Stokes wave given by (12) and (13).

\section{Bifurcation of the Stokes wave}

Steady solutions satisfy

$$
M(\mathbf{u}, C)=0 .
$$

Suppose that $s$ denotes arc-length along a continuous branch of solutions in the infinite dimensional space $(\mathbf{u}, C)$. Along the branch

$$
M^{\prime} \equiv M_{\mathbf{u}} \mathbf{u}^{\prime}+M_{C} C^{\prime}=0
$$

where ${ }^{\prime} \equiv d / d s$, and the suffices denote (Fréchet) derivatives of the operator $M$. If the homogeneous equation

$$
M_{\mathrm{u}} \Psi=0
$$

has a non-trivial solution, then the branch has either a limit point or bifurcates into a new type of steady profile. We shall now show that for arbitrarily given $\mathbf{p},(22)$ has solutions along the branch of Stokes waves (i.e. solutions in which the only non-zero components of $\mathbf{u}$ are those with $\mathbf{m}=0$ ), which do not correspond to limit points, at critical wave heights which depend on $\mathbf{p}$. Thus the Zakharov equation predicts that two-dimensional water waves of permanent form are infinitely degenerate to bifurcation into three-dimensional waves of permanent form. It is to be noted that the Zakharov equation is quantitatively accurate when the wave amplitude is small and the modulations are of long wavelength, i.e. $p^{2}+q^{2} \ll 1$. It will be seen that the critical wave height for bifurcation goes to zero as $p / q \rightarrow 0$. Hence, the results are quantitatively accurate for water waves in the limit of very oblique, long-wave modulations.

We can write (21) in the form

where

$$
\begin{aligned}
& M_{\mathrm{m}}^{\prime} \equiv G_{\mathrm{m}} a_{\mathrm{m}}^{\prime}+\sum T_{\mathrm{m}, \mathbf{i}, \mathbf{j}, \mathbf{k}}\left(\hat{a}_{\mathrm{i}}^{\prime} a_{\mathrm{j}} a_{\mathbf{k}}+\hat{a}_{\mathrm{i}} a_{\mathrm{j}}^{\prime} a_{\mathrm{k}}+\hat{a}_{\mathrm{i}} a_{\mathrm{j}} a_{\mathbf{k}}^{\prime}\right)-C^{\prime}(1+m p) a_{\mathrm{m}}=0, \\
& \widehat{M}_{\mathrm{m}}^{\prime} \equiv-G_{\mathrm{m}} \hat{a}_{\mathrm{m}}^{\prime}-\sum T_{\mathrm{m}, \mathrm{i}, \mathrm{j}, \mathrm{k}}\left(a_{\mathrm{i}}^{\prime} \hat{a}_{\mathbf{j}} \hat{a}_{\mathbf{k}}+a_{\mathrm{i}} \hat{a}_{\mathrm{j}}^{\prime} \hat{a}_{\mathbf{k}}+a_{\mathrm{i}} \hat{a}_{\mathrm{j}} \hat{a}_{\mathbf{k}}^{\prime}\right)+C^{\prime}(1+m p) \hat{a}_{\mathrm{m}}=0,
\end{aligned}
$$

$$
G_{\mathrm{m}}=\left[(1+m p)^{2}+n^{2} q^{2}\right]^{\frac{1}{4}}-C(1+m p) .
$$

Then (22) has a non-trivial solution on the Stokes wave branch, if the equations

$$
\begin{gathered}
\left(G_{\mathrm{m}}+2 a^{2} T_{\mathrm{m}, 0, \mathrm{~m}, 0}\right) a_{\mathrm{m}}^{\prime}+a^{2} T_{\mathrm{m},-\mathrm{m}, 0,0} \hat{a}_{-\mathrm{m}}^{\prime}=0, \\
-\left(G_{-\mathrm{m}}+2 a^{2} T_{-\mathrm{m}, 0,-\mathrm{m}, 0}\right) \hat{a}_{-\mathrm{m}}^{\prime}-a^{2} T_{-\mathrm{m}, \mathrm{m}, 0,0} a_{\mathrm{m}}^{\prime}=0,
\end{gathered}
$$

have non-trivial solutions. The necessary and sufficient condition is

$$
\left|\begin{array}{ll}
G_{\mathrm{m}}+2 a^{2} T_{\mathrm{m}, 0, \mathrm{~m}, 0}, & a^{2} T_{\mathrm{m},-\mathrm{m}, 0,0} \\
-a^{2} T_{-\mathrm{m}, \mathrm{m}, 0,0}, & -G_{-\mathrm{m}}-2 a^{2} T_{-\mathrm{m}, 0,-\mathrm{m}, 0}
\end{array}\right|=0,
$$


where without loss of generality we can take $m=( \pm 1, \pm 1)$. With the substitution of (13) for $C$, (26) becomes a quadratic for $a^{2}$ whose solution gives the critical wave height. It will be shown below that this equation has at least one positive root if $q / p$ is not too large. Moreover, the root is the same for each of the four values of $\mathbf{m}$.

Then, for given values of $p$ and $q$, solutions of the system (23) exist with

$$
\begin{array}{cl}
a_{\mathrm{m}}^{\prime}=\hat{a}_{\mathrm{m}}^{\prime}=\alpha \lambda_{\mathrm{m}}, & \hat{a}_{-\mathrm{m}}^{\prime}=a_{-\mathrm{m}}^{\prime}=\alpha, \quad \text { when } \quad \mathbf{m}=(1,1), \\
a_{\mathrm{m}}^{\prime}=\hat{a}_{\mathrm{m}}^{\prime}=\beta \lambda_{\mathrm{m}}, & a_{-\mathrm{m}}^{\prime}=a_{-\mathrm{m}}^{\prime}=\beta, \text { when } \quad \mathbf{m}=(1,-1), \\
a_{0}^{\prime}=\hat{a}_{0}^{\prime}=\frac{a C^{\prime}}{G_{0}+3 T_{0,0,0,0}}
\end{array}
$$

and $a_{\mathrm{m}}^{\prime}=0$ otherwise, where

$$
\lambda_{\mathrm{m}}=\frac{-T_{\mathrm{m},-\mathrm{m}, 0,0} a^{2}}{G_{\mathrm{m}}+2 a^{2} T_{\mathrm{m}, 0, \mathrm{~m}, 0}}=1 / \lambda_{-\mathrm{m}} .
$$

If $\alpha$ and $\beta$ are zero, $\dagger(28)$ gives the rate of change of $a$ along the branch of Stokes waves, as it is identical with the expression obtained by differentiating (13). The possibility of non-zero $\alpha$ and $\beta$ indicates the existence of a bifurcation at a critical amplitude $a$ which is a root of (26). One relation between $\alpha, \beta$ and $C^{\prime}$ is provided by the normalization condition

$$
\left\|\mathbf{u}^{\prime}\right\|^{2}+C^{\prime 2}=1
$$

but this essentially only fixes the arc length $s$. To find significant relations, we must consider the higher derivatives of $M$. From the second derivative evaluated at the critical wave height, we obtain

$$
M_{\mathrm{m}}^{\prime \prime} \equiv-2 C^{\prime} a_{\mathrm{m}}^{\prime}+2 a \sum\left\{2 T_{\mathrm{m}, \mathrm{i}, \mathrm{j}, 0} \hat{a}_{\mathrm{i}}^{\prime} a_{\mathrm{j}}^{\prime}+T_{\mathrm{m}, \mathbf{0}, \mathbf{j}, \mathbf{k}} a_{\mathrm{j}}^{\prime} a_{\mathbf{k}}^{\prime}\right\}+\left.M_{\mathrm{m}}^{\prime}\right|_{\substack{a_{\mathrm{m}}^{\prime} \rightarrow a_{\mathrm{m}}^{\prime \prime} \\ C^{\prime} \rightarrow C^{\prime \prime}}}=0
$$

and a similar equation for $\hat{M}_{\mathrm{m}}^{\prime \prime}$ by interchanging $a_{\mathrm{m}}$ and $\hat{a}_{\mathrm{m}}$ and changing the sign. The last term denotes (23a) evaluated with $a_{\mathrm{m}}^{\prime \prime}$ and $C^{\prime \prime}$ replacing $a_{\mathrm{m}}^{\prime}$ and $C^{\prime}$. The solubility condition (Fredholm alternative) of the inhomogeneous equations for $a_{m}^{\prime \prime}$, $\hat{a}_{\mathrm{m}}^{\prime \prime}$ and $C^{\prime \prime}$ gives relations between $\alpha, \beta$ and $C^{\prime}$. There are two equations, one arising from $\mathbf{m}=(1,1)$, and the other from $\mathbf{m}=(1,-1)$. Substitution of $(27)$ and $(28)$ into (31), and elimination of the terms in $a_{\mathrm{m}}^{\prime \prime}, \widehat{a}_{\mathrm{m}}^{\prime \prime}$ and $C^{\prime \prime}$ gives equations which reduce, respectively, to

Thus, either

$$
\alpha C^{\prime}=0, \quad \beta C^{\prime}=0 .
$$

or

$$
\alpha=\beta=0, \quad C^{\prime}=1
$$

$$
C^{\prime}=0, \quad \alpha^{2}+\beta^{2}=\frac{1}{2\left(1+\lambda^{2}\right)}
$$

[using the normalization condition (30)] where the ratio $r=\alpha / \beta$ is so far arbitrary.

Clearly (33) describes continuation along the Stokes wave branch. But (34) gives a new branch of three-dimensional solutions, in which

$$
\left.\begin{array}{l}
a_{0}=a, \quad C=1+T_{0,0,0,0} a^{2} \\
a_{(1,1)}=\epsilon \lambda_{(1,1)}, \quad a_{(-1,-1)}=\epsilon \\
a_{(1,-1)}=r \epsilon \lambda_{(1,-1)}, \quad a_{(-1,1)}=r \epsilon
\end{array}\right\}
$$

$\dagger$ The numbers $\alpha$ and $\beta$ can be supposed real with no loss of generality - this is just equivalent to an appropriate choice of phase or origin of $y$. 
correct to order $\epsilon$. These describe three-dimensional waves of permanent form, in which the surface elevation is

$$
\begin{aligned}
\eta= & \frac{1}{\pi \sqrt{ } 2} a \cos (x-C t) \\
& +\epsilon \lambda_{(1,1)}\left[(1-p)^{2}+q^{2}\right]^{\frac{1}{8}} \cos \{(1+p)(x-C t)+q y\} \\
& +\epsilon\left[(1-p)^{2}+q^{2}\right]^{\frac{1}{8}} \cos \{(1-p)(x-C t)-q y\} \\
& +r \epsilon \lambda_{(1,-1)}\left[(1+p)^{2}+q^{2}\right]^{\frac{1}{8}} \cos \{(1+p)(x-C t)-q y\} \\
& \left.+r \epsilon\left[(1-p)^{2}+q^{2}\right]^{\frac{1}{8}} \cos \{(1-p)(x-C t)+q y)\right\}+O\left(\epsilon^{2}\right) .
\end{aligned}
$$

The order $\epsilon^{2}$ terms on a branch can be found by studying $M^{\prime \prime \prime}=0$, and so on, but the algebra quickly becomes too involved to handle easily. It proves easier to follow the branch by solving numerically for steady solutions of a truncated version of (15). A truncation in which the integers range from $-N$ to $N$ is equivalent to calculating the $(N-1)$ th-order derivative of $M(\mathbf{u}, C)$ or finding the coefficient of $\epsilon^{N-1}$. Note that the coefficients of the waves which start branching as described by (35) are all real.

However, one condition can be quickly established and that is that $r=0$ or 1 . In other words, the wave is either completely skewed or symmetrical. (The case $r=\infty$ is the same as $r=0$ with the $y$ direction reversed.) This follows from noting that the Fredholm alternative takes the form

where

$$
\mu_{\mathrm{m}} M_{\mathrm{m}}^{\prime}+\hat{M}_{-\mathbf{m}}^{\prime}=0, \quad \mathrm{~m}=( \pm 1, \pm 1)
$$

$$
\mu_{\mathrm{m}}=\frac{a^{2} T_{-\mathrm{m}, \mathrm{m}, 0,0}}{G_{\mathrm{m}}+2 a^{2} T_{\mathrm{m}, 0, \mathrm{~m}, 0}}=1 / \mu_{-\mathrm{m}}
$$

Applying the Fredholm alternative to $M^{\prime \prime \prime}$ for $\mathbf{m}=(1,1)$ and $\mathbf{m}=(1,-1)$ gives equations for $C^{\prime \prime}$ which are inconsistent unless $r=0$ or 1 .

It is to be noted that if $a_{\mathrm{m}}$ describes a steady solution, so does $a_{\mathbf{m}} \exp [i(\gamma+\mathbf{m} . \nu)]$ where $\gamma$ and $\nu$ are real constants. This trivial degeneracy is due to gauge and Galilean invariances, and can be eliminated in the calculation of steady solutions by requiring that $a_{0}$ and $a_{(1, \pm 1)}$, say, are real. However, it does have significant consequences when we consider the stability of the bifurcated solution in a later section.

The critical wave heights $a$ at which bifurcations occur depend on $\mathbf{p}=(p, q)$. In figure 1 , we show wave steepness $2^{-\frac{1}{2}} a / \pi$ as a function of $q$ for various values of $p$. The case $q=0$ corresponds to two-dimensional bifurcation of the type considered by Chen \& Saffman (1980) and Saffman (1980), who studied the full water-wave equation. It is interesting to note that the results agree qualitatively even though they lie outside of the apparent range of validity of the Zakharov approximation, e.g. the decrease of the critical amplitude with $p$. The quantitative values of critical wave steepness are about $50 \%$ too high for the two-dimensional case.

In figure 2 , we plot $2^{-\frac{1}{2}} a / \pi$ against $\theta=\tan ^{-1}(q / p)$, which is a measure of the degree of obliqueness of the three-dimensional modulation for various values of $p$. Also shown is the curve for neutral stability of a uniform wave train to three-dimensional disturbance of infinite wavelength calculated from Whitham's exact finite-amplitude theory (see Peregrine \& Thomas (1979) and Saffman (1980) for details). It is interesting to note that for $2^{-\frac{1}{2}} a / \pi$ less than $0 \cdot 35$, the difference between the curve with $p=10^{-5}$ and Whitham's result (which is exact in the limit of $p, q \rightarrow 0$ ) is practically undetect- 


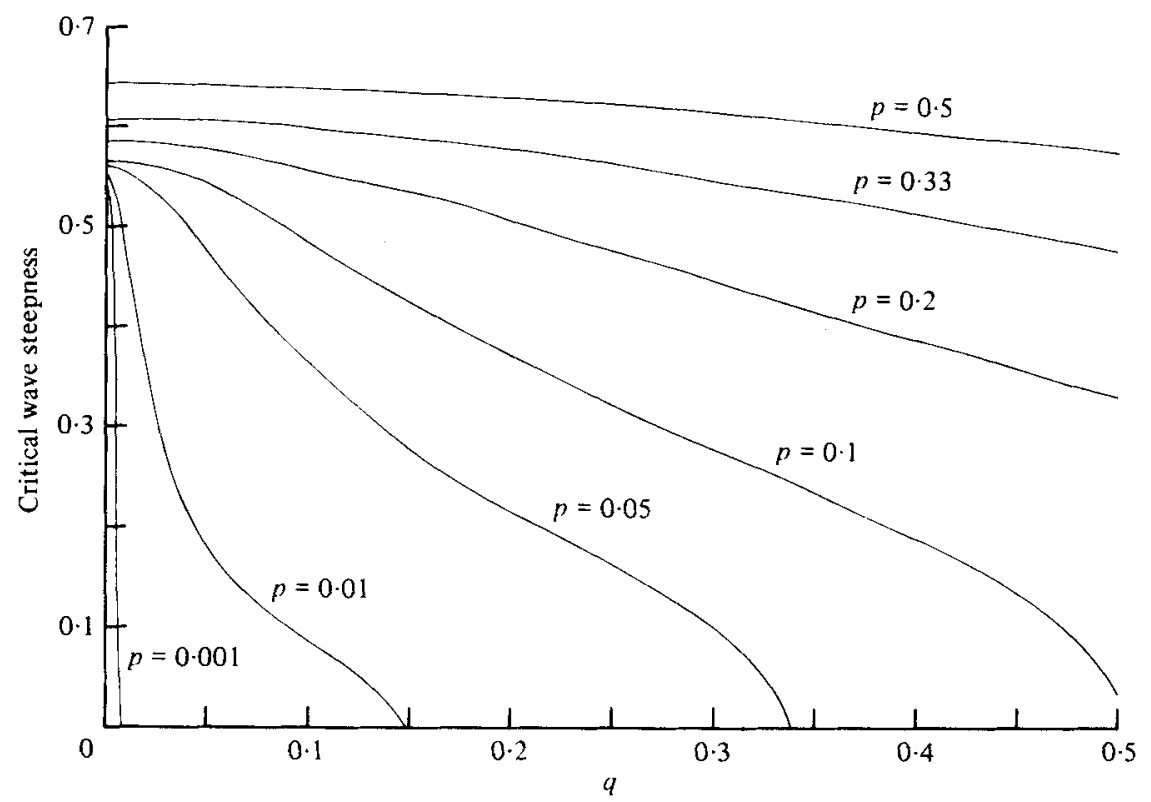

Frgure 1. Critical wave steepness $2^{-\frac{1}{2}} a / \pi$ as a function of $q$ for various values of $p$.

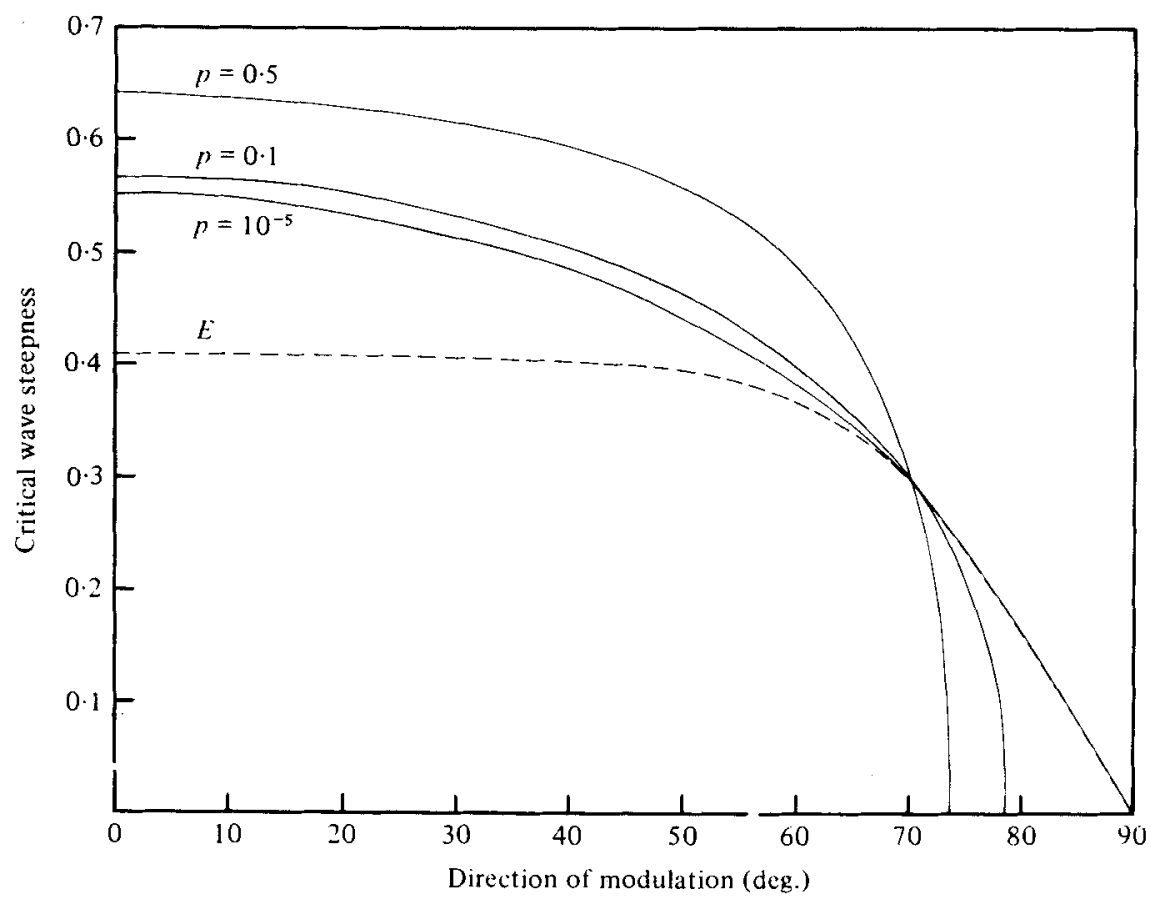

FIGURe 2. Critical wave steepness as a function of direction of modulation measured by the angle in degrees between the modulational wave vector $\mathbf{p}$ and the direction of propagation of the unbifurcated solution $\mathbf{e}_{1}$. Curve marked $E$ represents the neutral stability curve obtained from Whitham's theory which is exact in the limit of $p, q$ approaching zero. 
able. This is a strong suggestion that the present results are quite accurate for wave steepness $2^{-\frac{1}{2}} a / \pi$ less than $0 \cdot 35$ (which covers about $80 \%$ of the permissible range of water wave steepness) and $\theta$ more than $60^{\circ}$.

\section{Properties of skew waves}

We now consider the question of following the family of three-dimensional waves a finite distance along the branch. The bifurcation is degenerate because the parameter $r$ can be either 0 or 1 . The case $r=1$ gives three-dimensional waves which are symmetrical about the direction of propagation. For $r=0$, the pattern is skewed.

The calculation is somewhat simpler for $r=0$, and we shall, in the present paper, confine ourselves to the discussion of this case. When $r=0$, the wavenumbers that are generated by the higher-order interactions are all of the form

$$
\mathbf{1}=\mathbf{e}_{1}+m(1,1)
$$

and can be characterized therefore by a single integer $m,-\infty<m<\infty$. Also, the amplitudes are all real. Writing then

$$
\hat{a}_{\mathrm{m}}=a_{\mathrm{m}}=b_{m}
$$

and with an obvious change of notation for the interaction coefficients, the skew waves are given by the solutions of the equations

$$
\begin{aligned}
{\left[(1+m p)^{2}+m^{2} q^{2}\right]^{\ddagger} b_{m}-C(1+m p) b_{m}+\sum_{m=i=j+k} T_{m, i, j, k} b_{i} b_{j} b_{k}=0, } & -\infty<m<\infty .
\end{aligned}
$$

We have solved truncated forms of these equations by Newton's method, generating new solutions by Euler continuation. We use $b_{1}$ as a given parameter and then solve the $2 N+1$ equations for $b_{m}(-N \leqslant m \leqslant N)$ and $C$ as functions of $b_{1}$. As

$$
b_{1} \rightarrow 0, \quad b_{-1} \rightarrow b_{1} / \lambda_{(1,1)}, \quad b_{0} \rightarrow a, \quad C \rightarrow 1+T_{0,0,0,0} a^{2}
$$

and all other coefficients are $o\left(b_{1}\right)$. In figure 3 , we show plots of $b_{0}$ and $C$ as functions of $b_{1}$ on the bifurcated branch.

In figure 4, we show examples of the free surface of the bifurcated three-dimensional steady solution for the case $p=0 \cdot 1, q=0 \cdot 4$. It is interesting to note that recent experimental observations by Su (private communications) strongly suggest the existence of these bifurcated steady waves in nature.

\section{Stability of skew waves to infinitesimal perturbations}

The stability of the waves to infinitesimal perturbations is a matter of considerable interest. Suppose that $\mathbf{u}, C$ is a steady solution of (20). We consider an infinitesimal perturbation of the form $\mathbf{u}+\mathbf{v} e^{i \sigma t}$ where $\|\mathbf{v}\| \ll\|\mathbf{u}\|$. Then from (17), v satisfies the equation

where

$$
-\sigma \mathbf{v}=M_{\mathbf{u}}(\mathbf{u}, C) \mathbf{v}
$$

$$
\mathbf{v}=\left(\left\{v_{m}\right\} ;\left\{\hat{v}_{m}\right\}\right)
$$




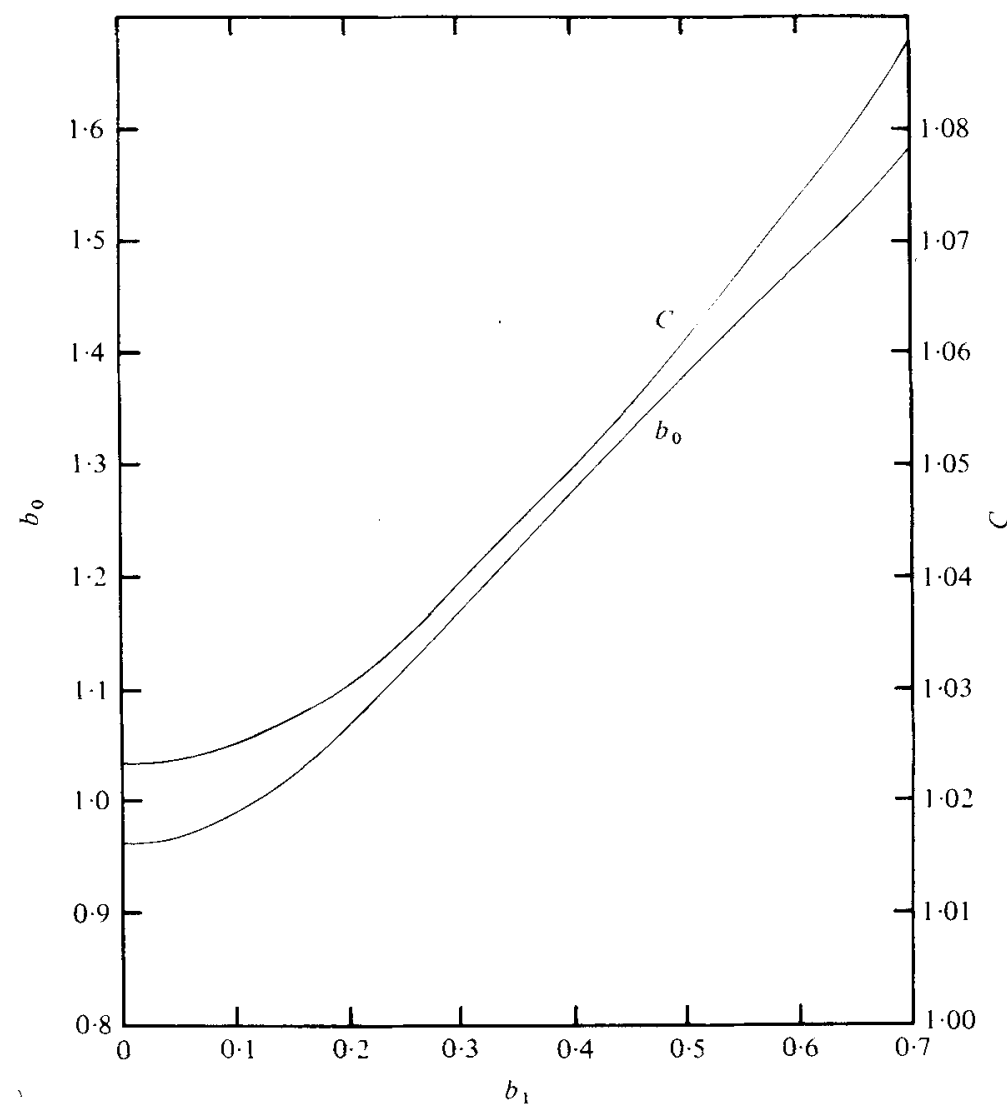

Figure 3. Plots of $b_{0}$ and $C$ as functions of $b_{1}$ on the bifurcated branch. The case is for $p=0.05$ and $q=0 \cdot 2$. Note that $b_{0} \rightarrow a$ and $C \rightarrow 1+T_{0,0,0,0} a^{2}$ as $b_{1} \rightarrow 0$.

and

$$
\begin{aligned}
& \left\{\sigma+\left[(1+m p)^{2}+n^{2} q^{2}\right]^{\ddagger}-C(1+m p)\right\} v_{\mathrm{m}}+2 \sum T_{\mathrm{m}, \mathrm{i}, \mathrm{j}, \mathrm{k}} a_{\mathrm{i}}^{*} a_{\mathrm{j}} v_{\mathrm{k}} \\
& +\sum T_{\mathrm{m}, \mathbf{k}, \mathrm{i}, \mathrm{j}} a_{\mathrm{i}} a_{\mathrm{j}} \hat{v}_{\mathbf{k}}=0, \\
& \left\{\sigma-\left[(1+m p)^{2}+n^{2} q^{2}\right]^{4}+C(1+m p)\right\} \hat{v}_{\mathrm{m}}-2 \sum T_{\mathrm{m}, \mathrm{i}, \mathrm{j}, \mathbf{k}} a_{\mathbf{i}} a_{\mathbf{j}}^{*} \hat{v}_{\mathbf{k}} \\
& -\sum T_{\mathrm{m}, \mathbf{k}, \mathbf{i}, \mathbf{j}} a_{\mathbf{i}}^{*} a_{\mathrm{j}}^{*} v_{\mathbf{k}}=0 \text {. }
\end{aligned}
$$

These equations constitute an eigenvalue problem for $\sigma$ and the associated eigenfunction $\mathbf{v}$. Note that $\mathbf{v}$ need not satisfy the condition (16), because if $\sigma$ and $\mathbf{v}$ give a solution, so do $\sigma^{*}$ and $\mathbf{v}^{*}$. Also $\mathbf{v}^{R} \equiv\left(\left\{\hat{v}_{m}\right\} ;\left\{v_{m}\right\}\right)$ is a solution with $\sigma$ replaced by $-\sigma$. Hence $\mathrm{v} e^{i \sigma t}+\left(\mathrm{v}^{R}\right)^{*} e^{-i \sigma^{*} t}$ will generate solutions of (42) which satisfy (16). Our task is therefore to investigate the properties of the eigenvalue problem (44) where the coefficients describe steady waves of permanent form.

The bifurcation point given by (26) is a point of neutral stability $(\sigma=0)$ for disturbances described by the modulation wave vector $\mathbf{p}$ and $\mathbf{m}=(1,1)$ or $\mathbf{m}=(1,-1)$. If we follow the Stokes wave through the bifurcation point, it is easily shown that $\sigma$ stays real for these disturbances; i.e., the bifurcation point is not a point of stability exchange. Our interest lies in the behaviour of the vanishing eigenvalues as we follow 

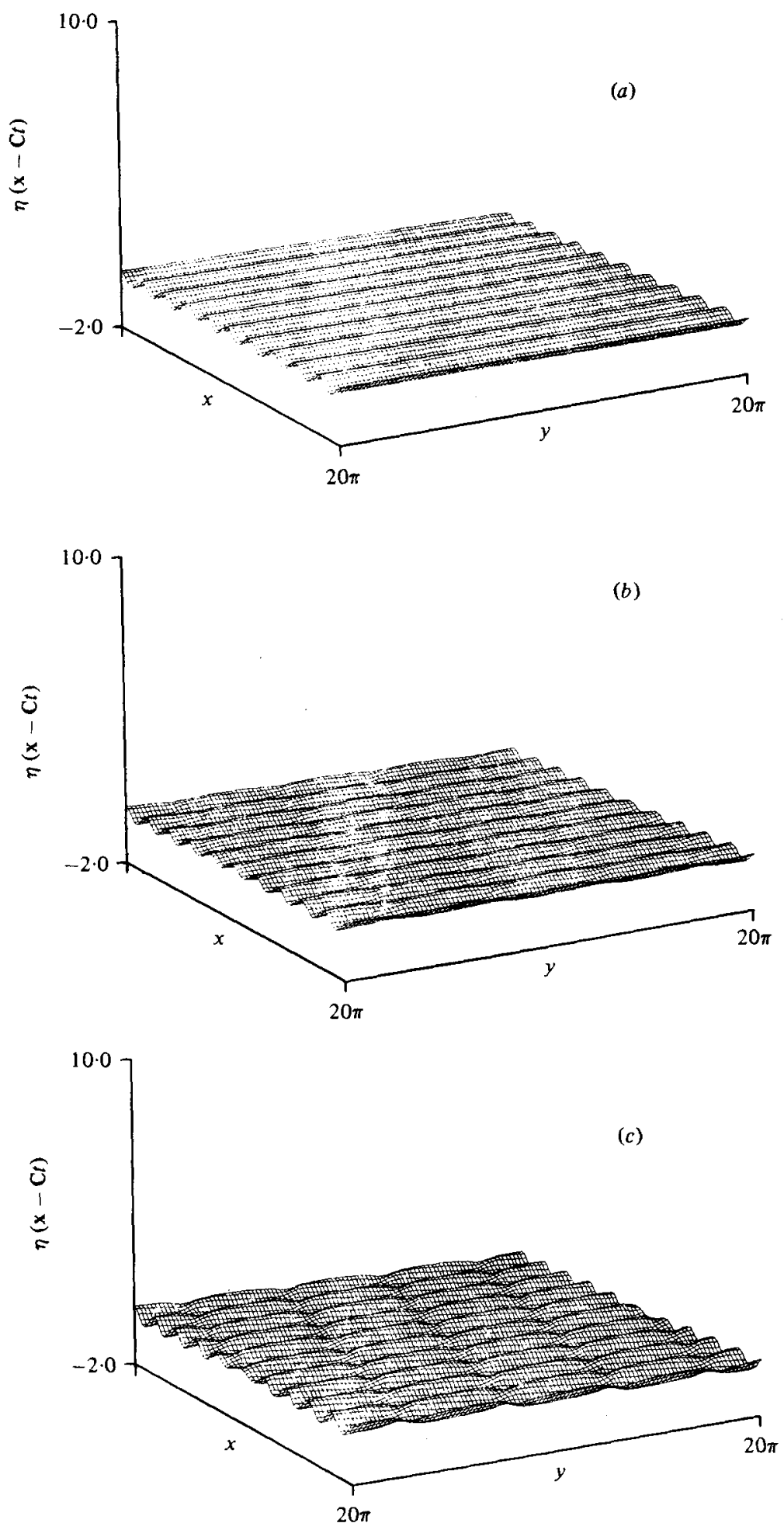

FIaUre 4. Free surface of water corresponding to bifurcated three-dimensional steady skew waves for the case $p=0 \cdot 1, q=0 \cdot 4$. (a) Unbifurcated state: uniform two-dimensional wavetrain; (b) bifurcated skew waves, $b_{1}=0.2 ;(c)$ bifurcated skew waves, $b_{1}=0.4$. Note that the vertical scale has been expanded for the purpose of illustration. 


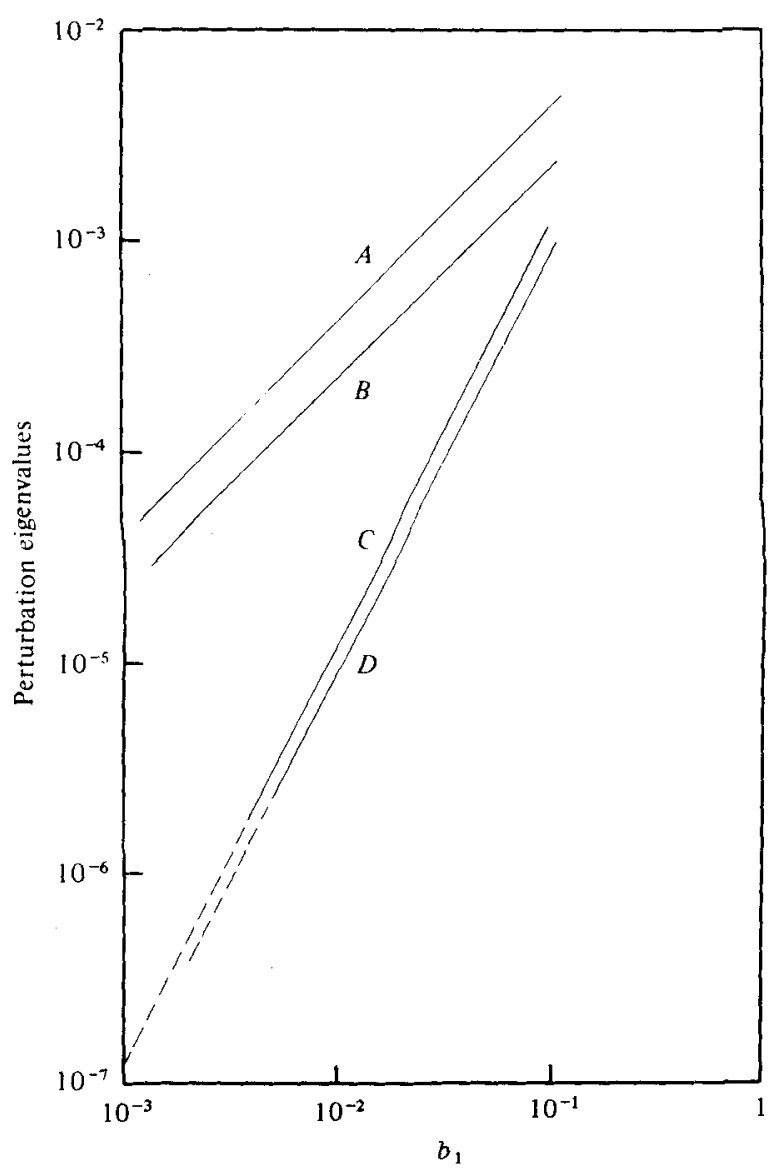

Fraure 5. Perturbation eigenvalues $\sigma$ as functions of $b_{1}$. Curves $A$ and $B$ correspond to the gauge invariance vector $\mathbf{v}^{(1)}$; note the linear dependence on $b_{1}$. Curves $C$ and $D$ correspond to the null eigenvectors $\mathrm{v}^{(3)}$ and $\mathrm{v}^{(3) R}$; note the quadratic dependence on $b_{1}$. Curves $A$ and $C$ are for the case $p=0.05, q=0.2$; curves $B$ and $D$ are for the case $p=0.1, q=0.4$.

the new branches and here we investigate their behaviour along the branch of skew waves calculated in the last section. For these waves, $a_{\mathbf{m}}=b_{m}, \mathbf{m}=m(1,1)$. There are two classes of perturbations $\mathbf{v}$. Those for which $\mathbf{m}=m(1,1)$, and those with

$$
\mathbf{m}=m(1,-1) \text {. }
$$

We dispose first of the former type. The eigenvalue problem (44) can be written in block matrix form with an obvious notation as

$$
\left(\begin{array}{cc}
A & B \\
-B & -A
\end{array}\right)\left(\begin{array}{l}
v \\
\hat{v}
\end{array}\right)+\sigma\left(\begin{array}{l}
v \\
\hat{v}
\end{array}\right)=0
$$

Because of the gauge and Galilean invariance, there are two null eigen-vectors $\mathbf{v}^{(1)}=\left(\left\{b_{m}\right\},\left\{-b_{m}\right\}\right)$, and $\mathbf{v}^{(2)}=\left(\left\{m b_{m}\right\},\left\{-m b_{m}\right\}\right)$. Each eigenvector corresponds to a double zero, as they are orthogonal to their adjoints, which are easily seen to be $\mathbf{v}^{(1) t}=\left(\left\{b_{m}\right\},\left\{b_{m}\right\}\right)$ and $\mathbf{v}^{(2) t}=\left(\left\{m b_{m}\right\},\left\{m b_{m}\right\}\right)$. The eigenvector $\mathbf{v}^{(2)}$ exists only on the skew wave branch. Thus the transition from Stokes wave to skew wave is characterized 
by the appearance of a second null eigenvector associated with the new branch. There is therefore no change of stability with respect to disturbances whose dominant terms near the bifurcation point are those with $\mathbf{m}= \pm(1,1)$.

We now consider the disturbances with dominant modes $\mathbf{m}= \pm(1,-1)$. In this case, the suffices in (44) are such that $\mathbf{m}$ and $\mathbf{k}$ are proportional to $(1,-1)$, and $\mathbf{i}$ and $\mathbf{j}$ are proportional to $(1,1)$. It is again possible to study the behaviour of the eigenvalues analytically. The null eigenvectors at the bifurcation point are $\mathbf{v}^{(1)}, \mathbf{v}^{(3)}$ and $\mathbf{v}^{(3) R}$, where

$$
\mathbf{v}^{(3)}=\left(\left\{0,0,0, \lambda_{(1,-1)}, 0\right\} ;\{0,1,0,0,0\}\right) .
$$

It follows from the analysis that the four eigenvalues are real (i.e. stable) and non zero as the new branch is followed. They appear in pairs of equal magnitude and opposite sign. Those associated with $\mathbf{v}^{(1)}$ are initially proportional to $b_{1}$ and those associated with $\mathbf{v}^{(3)}$ and $\mathbf{v}^{(3) R}$ are proportional to $b_{1}^{2}$. The analysis is fairly involved and not altogether straightforward, but is not given here because for the actual computation of the eigenvalues, it proved easier to solve the eigenvalue problem directly. In figure 5, we show a plot of the eigenvalues as a function of $b_{1}$ to illustrate the abovementioned dependence.

We wish to thank Professor Bengt Fornberg for his advance and assistance in the choice and implementation of the numerical methods.

\section{REFERENCES}

Chen, B. \& Safrman, P. G. 1980 Numerical evidence for the existence of new types of gravity waves of permanent form on deep water. Stud. Appl. Math, 62, 1-21.

Crawford, D. R., Lake, B. M., Saffman, P. G. \& Yuen, H. C. 1981 Stability of weakly nonlinear deep-water waves in two and three dimensions. $J$. Fluid Mech. (to appear).

Crawford, D. R., Saffman, P. G. \& Yuen, H. C. 1980 Evolution of a random inhomogeneous field of nonlinear deep-water gravity waves. Wave Motion 2, 1-16.

Peregrine, D. H. \& Thomas, G. P. 1979 Finite amplitude deep water waves on currents. Phil. Trans. Roy. Soc. A 292, 371-390.

SaffMan, P. G. 1980 Long wavelength bifureation of gravity waves on deep water. $J$. Fluid Mech. 101, 631-647.

Schwartz, L. W. 1974 Computer extension and analytic continuation of Stokes expansion for gravity waves. J. Fluid Mech. 62, 553-478.

STokes, G. G. 1847 On the theory of ascillatory waves. Camb. Trans. 8, 441-473 (Papers, vol. I, pp. 197-229).

Zakharov, V. E. 1968 Stability of periodic waves of finite amplitude on the surface of a deep fluid. J. Appl. Mech. Tech. Phys. 2, 190-194. 